Christian Rüdiger

\title{
Gaudeamus igitur
}

\author{
Poetische Weltentfaltung und ästhetische Weltwahrnehmung \\ als zeithistorische Dimensionen in Werner Jacobs' ZUR HöLLE \\ MIT DEN PAUKERN
}

\begin{abstract}
Ein Wort noch zu der Serie der Lümmel- und Paukerfilme, mit denen Papas Kino ausgangs der sechziger Jahre seinen kläglichen Abgesang zu feiern begann. Werner Jacobs' DIE LÜMMEL VON DER ERSTEN BANK - ZUR HÖLLE MIT DEN PAUKERN; Harald Reinls PEPE, DER PAUKERSCHRECK (BRD 1969); Werner Jacobs' ZUM TEUFEL MIT DER PENNE; HURRA, DIE SCHULE BRENNT! (BRD 1969) vom selben Regisseur; WIR HAU'N DIE PAUKER IN DIE PFANNE (BRD 1970), wiederum von Harald Reinl; Harald Volcks UNSERE PAUKER GEHEN IN DIE LUFT (BRD 1970) und wie die weiteren Klamotten dieser Serie alle heißen, wurden nach einheitlichem Strickmuster heruntergedreht. Wer sich als „normaler“ Kinobesucher nur drei von diesen Klamauk-Werken angetan hat, dürfte nach kurzer Zeit Schwierigkeiten haben, die verschiedenen Filme auseinanderzuhalten. Die Titel waren meist aggressiv. Dennoch hatten die Filme nichts mit der antiautoritären Schüler- oder Studentenbewegung im Sinn. ${ }^{1}$
\end{abstract}

Für Friedrich Koch, der diese Worte Ende der 1980er Jahre fand, sind diese Streifen nicht mehr als der schnoddrige Bodensatz am Grunde von Papas zu Recht dahinsiechendem Kino, mit dem es sich höchstens $\mathrm{zu}$ beschäftigen lohnt, wenn man auf die Wegwerfqualität dieser ,filmischen Einmalprodukte، hinweisen möchte.

Wenn man sich die Mühe macht, diese Filme anzusehen, so wird man feststellen, dass sie durchaus divers sind und Potential für historisch motivierte Reflexion bieten. Dieser Essay setzt sich zum Ziel, einige Aspekte der Weltentfaltung und -wahrnehmung des ersten Films der Reihe etwas näher zu beleuchten, also die Frage zu stellen, was das überhaupt für eine Welt ist, die hier entworfen wird, wie sich diese uns öffnet und wie sie sich in den filmhistorischen Kontext einbetten lässt. Dafür werde ich die episodische Struktur des Films selbst, wie auch der Filmreihe untersuchen und Fragen an die Inszenierung der Körper richten, um letztlich die Weltenfaltung der Lümmelfilme als eine spezifische, raumzeitliche Konfiguration bestimmen zu können.

1 Friedrich Koch: Schule im Kino. Autorität und Erziehung. Vom ,Blauen Engel' bis zur ,Feuerzangenbowle‘. Weinheim/Basel 1987, S. 187-188. 


\section{Episodenhaftigkeit im Film}

Versucht man den Inhalt von ZUR HÖLLE MIT DEN PAUKERN ${ }^{2}$ zusammenzufassen, fällt zunächst sein episodischer Aufbau auf. Hierin spiegelt sich die Struktur der unter dem Pseudonym Alexander Wolf verfassten literarischen Vorlage von Herbert Rösler (1963) wider: Meist nur wenige Seiten lange, untereinander kaum verbundene und mit satirischem Sprachwitz gespickte, anekdotenhafte Streiche und Kuriositäten aus dem Schulleben. Ein Großteil des Films, der sich auf einige Stellen des Buches teilweise recht detailgetreu bezieht, ist durch ebendiese Episodenhaftigkeit gekennzeichnet, die über weite Strecken eine revueartige Aneinanderreihung von Slapstickeinlagen und körperbetonter Situationskomik produziert. Neben dem Verweis auf Röslers Buch lohnt sich jedoch auch der Hinweis auf Filme aus dem Bereich der Posse und des Schwankes, die, wie Hans J. Wulff beschreibt, ebenfalls oft episodisch und von derbem, auch Körperfunktionen betreffenden, Humor gekennzeichnet sind. ${ }^{3}$

Im Grunde genommen enthält ZUR HÖLLE MIT DEN PAUKERN drei sich teilweise überschneidende Handlungsebenen: 1 . die nur lose miteinander und dem Rest des Films verbundenen Streiche; 2. die Begebenheiten um die von Pepes Vater umworbene französische Austauschschülerin Geneviève Ponelle; und 3. die Liebesgeschichte zwischen dem neuen und progressiv auftretenden Lehrer Dr. Albert Kersten und der studierenden Tochter (Helena) des Oberstudiendirektors Dr. Taft. Erst zum Ende des Films finden die bis zu diesem Zeitpunkt mehr oder weniger parallellaufenden Stränge auf der Ebene der Liebesbeziehung zusammen - wenn auch wiederum eher lose.

Auch spätere Einträge der filmischen Reihe um den Schüler Pepe Nietnagel sind von einer ähnlichen Struktur aus Streichen und ,Handlung' gekennzeichnet, obwohl sie mit einer stärkeren Fokussierung auf zentrale Figuren weniger parallellaufende Stränge entwickeln. Zusammen mit den vielseitigen Ablegern und Nachahmern bilden sie ein größeres mediales Konglomerat aus Kinofilmen, Schlagermusik und Populärkultur. Der erste Teil der Filmreihe erreichte

2 Auch: DIE LÜMMEL VON DER ERSTEN BANK 1. TEIL - ZUR HÖLLE MIT DEN PAUKERN.

3 Die Streiche fungieren hier als Auflockerungen, welche musikalischen Nummern gleich episodisch eingestreut werden, was für Wulff eher ein Charakteristikum der Posse als des Schwankes darstellt, welcher stärker auf dramaturgische Geschlossenheit setze. Gleichzeitig stärkt Wulff jedoch auch eine Position dynamischer Übergänge, welche es unpraktisch und unhandlich mache, zwischen „Klamotte (oft als ,derber Schwank ausgewiesen), Klamauk, Posse, Farce, Burleske [und, C.R.] Groteske“ zu unterscheiden. Vgl. Hans J. Wulff: Hybridität der Gattungen. Schlagerfil/Filmschwank/Schlagerfilmschwank. In: Hanno Berger et al. (Hg.): Prekäre Genres. Zur Ästhetik peripherer, apokrypher und liminaler Gattungen. Bielefeld 2015, S. 217235, hier S. 218. 
fast sechs Millionen Zuschauer, der zweite Teil zUM TEUfEL MIT DER PENNE mit Peter Alexander und Heintje (in einem Kurzauftritt) besetzt, sogar noch mehr. Unter den deutschen Musik-Top-10 des Jahres 1968 finden sich als einzige deutschsprachige Künstler immerhin zwei Einträge von Peter Alexander und drei von Heintje (der erfolgreichste Titel des Jahres, Heintjes Lied Mama, wird in ZUM TEUFEL MIT DER PENNE in voller Länge dargeboten). Beide Sänger spielen auch 1969 im vierten Teil der Reihe HURRA, DIE SCHULE BRENNT!, mit geschätzt vier Millionen Zuschauern. ${ }^{4}$ Auch wenn die Filme im ästhetischen Urteil nicht ganz unberechtigterweise $\mathrm{zu}$ den weniger interessanten der endsechziger Jahre zählen dürften, muss bereits hier Friedrich Kochs Eingangsbemerkung widersprochen werden. Etwas, das einen solchen ,Fußabdruck hinterlässt, muss es wert sein, untersucht zu werden.

\section{Episodenhaftigkeit im deutschen Nachkriegskino}

Das Prinzip, erfolgreiche deutsche Filme als Reihen oder in serieller Form hervorzubringen, ist jedoch nicht neu. Erfolgreich liefen Ende der 1950er, Anfang der 1960er Jahre, als sich die Krise des nach dem Krieg re-etablierten deutschen Kinos immer stärker abzuzeichnen begann, nur noch Filme auf der „Ebene des Trivialen“, wie die Edgar Wallace-, Karl May- und Jerry Cotten-Reihen. ${ }^{5}$

Es wird auf billig aufgemachte Illustriertengeschichten und banale Groschenromane zurückgegriffen, früher erfolgreiche Filme werden als Remakes neu produziert, und Filme mit überdurchschnittlichem Kassenerfolg gleich in Serie gegeben [...]. ${ }^{6}$

Was als Anklage an unser zeitgenössisches Mainstreamkino gedacht sein könnte, ist in der Tat der Vorwurf an das deutsche Kino der 1950er Jahre, stereotype Muster bis zum Exzess zu wiederholen - der Heimatfilm stellt hier das

4 o. V.: Jahrescharts Deutschland 1968. Auf: chartsurfer.de: http://www.chartsurfer.de/musik/ single-charts-deutschland/jahrescharts/hits-1968-2x1.html (27.04.2018), Joachim Kramp: Die Lümmel von der ersten Bank: Die Lümmel sind los! Auf: Luemmelbank.blogspot.de: http:// luemmelbank.blogspot.de/p/die-lummel-sind-los.html (27.03.2018) und o. V.: Die erfolgreichsten deutschen Filme seit 1963 (2018). Auf: insidekino.de: http://www.insidekino.com/DJahr/ DAlltimeDeutsch50.htm (27.04. 2018).

5 Norbert Grob: Film der sechziger Jahre. Abschied von den Eltern. In: Wolfgang Jacobson et al. (Hg.): Geschichte des deutschen Films. Stuttgart/Weimar 2004, S. 207-244, hier S. 210.

6 Gertraud Koch et al.: Die fünfziger Jahre. Heide und Silberwald. In: Wolfgang Kaschuba (Hg.): Der deutsche Heimatfilm. Bildwelten und Weltbilder - Bilder, Texte, Analysen zu 70 Jahren deutscher Filmgeschichte. Tübingen 1989, S. 69-95, hier S. 79. 
Standardbeispiel dar. ${ }^{7}$ Im Mainstreamkino der 1960er ändert sich daran praktisch nichts. Ende der 1950er Jahre zeichnete sich hingegen die zunehmende Dominanz des Fernsehens ab. ${ }^{8}$ Viele Kino-, Filmproduktions- und Verleihfirmen gingen bankrott (innerhalb eines Jahrzehnts schwanden die Zuschauerzahlen um fast $80 \%$ ) und die Zahl der produzierten Filme sank innerhalb von sechs Jahren bis 1962 um die Hälfte, während sich im selben Zeitraum die Anzahl der privaten Fernsehgeräte mit 7,2 Millionen mehr als verzehnfachte. ${ }^{9}$ In dieser Zeit veränderte sich die deutsche Filmproduktionslandschaft stark. Durch die zunehmende Zersplitterung der Produktions- und Vertriebslandschaft entstanden anstelle großer, prestigeträchtiger Produktionen einzelner Firmen zunehmend paneuropäische Koproduktionen kleinerer Studios. Die Zielgruppe der Produktionen veränderte sich nach Tim Bergfelder von weiblich und familiär zu jung, männlich und international; außerdem sieht er eine Verschiebung von ,frauenzentrierten“ Genres wie Melodrama und Heimatfilm zu Western, Abenteuergenres, Horror-, Kriminal- und Agententhrillern sowie SexFilmen - alle oft in seriellen Formaten. ${ }^{10}$ Edgar-Wallace-, Karl-May- und JerryCotton-Filme, wie auch die Lümmel- und Paukerfilme, machten Unternehmen wie Constantin und Gloria schließlich $\mathrm{zu}$ den erfolgreichsten deutschen Filmfirmen der 1960er. ${ }^{11}$

7 Obwohl der US-amerikanische Filmmarkt seit der Währungsreform 1948 massiv in Produktion, Distribution und Projektion investierte, blieben Hollywood-Filme lange Zeit eher nebensächlich. Vgl. Grob, Film der sechziger Jahre, S. 209 und vgl. Koch et al., Die fünfziger Jahre, S. 77-78. „Unter den jeweils zehn erfolgreichsten Filmen der Jahre 1950/51 bis 1962/63, insgesamt also 130 Streifen, waren 23 aus Hollywood; keiner von ihnen schaffte es auf den ersten Platz. Dem stehen 75 deutsche und 18 österreichische Erfolgsfilme gegenüber.“ Kaspar Maase: Was macht Populärkultur politisch? Wiesbaden 2010, S. 53.

8 Barbara Wilzcek argumentierte bereits Ende der 1980er gegen die allgemeine Behauptung der Kinobranche, das Fernsehen alleine wäre Schuld am Untergang des Kinos gewesen - man habe sich in der Monopolstellung im „audio-visuellen Freizeitbereich [...] offenbar zu sicher gewähnt“. Die Produzenten verkauften die Rechte ihrer Filme z. B. massenhaft und zu niedrigen Preisen an die Fernsehanstalten, wo sie dann mit wachsender Beliebtheit und unter gewaltigen Verlusten für die Produzenten liefen: „Die Senderechte eines Films werden für 20.000 bis 30.000 DM verkauft - die daraus entstehenden Einnahmeverluste belaufen sich jedoch auf Hunderttausende von DM.“ Koch et al.: Die fünfziger Jahre, S. 80.

9 Vgl. Grob: Film der sechziger Jahre, S. 216-217.

$10 \mathrm{Vgl}$. Tim Bergfelder: Exotic Thrills and Bedroom Manuals. West German B-Film Production in the 1960s. In: Randall Halle und Margaret McCarthy (Hg.): Light Movies. German Popular Film in Perspective. Detroit 2003, S. 197-219, hier S. 198.

11 Vgl. Bergfelder: Exotic Thrills, S. 197. Zum Ende der Dekade waren nur noch wenige der traditionellen deutschen Produktions- und Verleihfirmen im Geschäft, nach Bergfelder zum größten Teil abgelöst durch Produzenten und Verleiher von Exploitation-Filmen. Vgl. Bergfelder, Exotic Thrills, S. 199. 


\section{Episodenhaftigkeit zwischen Filmen}

Die Fortsetzungen der Lümmelfilme sind keine Sequels, wie wir sie im aktuellen Mainstreamkino finden. So knüpfen die sechs offiziellen Filme (bis 1972) inhaltlich in keiner Weise aneinander an. Einzig gemein ist ihnen ein relativ stabiles Darsteller- und Figurenensemble, welches jedoch selbst eher permutativen Charakter besitzt. ${ }^{12}$ Die Fortsetzungen sind mehr thematische Variationen oder Neukonstellationen voneinander. Auf diese Weise entstehen ambivalente und palimpsestartige Überschneidungen und Überschreibungen zwischen unterschiedlichen Filmen ähnlicher poetischer Verfasstheit. So nicht nur zwischen den Filmen der eigentlichen Reihe selbst, sondern auch zu ,Pauker- und Pennefilmen' anderer Produktionsfirmen, die nicht selten Teile des Ensembles, ähnliche Narrative, Streiche etc. teilen. Paradigmatisch ist hier Theo Lingen zu nennen, der in unterschiedlichsten Filmen als Oberstudiendirektor, Lehrer, Vater o.ä. auftritt und sich dabei praktisch immer am gleichen schauspielerischen Repertoire bedient.

ZUR HÖLLE MIT DEN PAUKERN steht dabei nur bedingt in der Tradition anderer Schulkomödien von Werner Jacobs aus den 1960ern wie DER MUSTERKNABE (BRD 1963), ... UND SOWAS MUSS UM 8 INS BETT (BRD 1965) oder DIE HEIDEN VON KUMMEROW UND IHRE LUSTIGEN STREICHE (BRD/DDR 1967). Der große Unterschied besteht in der prismatisch aufgebrochenen Struktur, welche die Episoden weder gänzlich parallel noch unabhängig voneinander oder tief miteinander vernetzt zeigt. Die einzelnen Stränge kreisen eher, durch schwache Beziehungen in der Bahn gehalten, um ein bis jetzt noch nicht näher definiertes, affektives Zentrum, welches sich sowohl innerhalb als auch außerhalb des Films befindet. Bevor ich versuche, dieses vage Zentrum näher zu verorten, muss ich zunächst auf einige weitere Aspekte des Films eingehen, die mir den Weg zum eigentlichen Punkt dieser Exploration erleichtern sollen.

\section{Liebe, Sex und Nazis - Von Körpern und ihren angenommenen Funktionen}

Der dramaturgische Dreh- und Angelpunkt von zUR HÖLLE MIT DEN PAUKERN wenn man dies überhaupt so klar sagen kann - ist die Liebesgeschichte zwi-

12 Peter Alexander taucht z. B. in zwei Filmen der Reihe in unterschiedlichen Rollen auf. Pepes Vater wird über die Filme von Georg Thomalla, Willy Millowitsch, Gustav Knuth, Wolfgang Gruner und Fritz Tillmann verkörpert. Ähnliches trifft auf die Lehrerinnen und sonstiges 
schen dem neuen Lehrer Dr. Kersten und Helena, der Tochter des Direktors, welcher mit seiner Familie im Schulgebäude wohnt und dort das verschrobene Ideal einer Bildungsbürgerfamilie des 19. Jahrhunderts lebt. Dabei entgleitet dem patriarchalen Oberhaupt die Kontrolle über seine ,flügge werdende‘, pixie cut und Hosen tragende Tochter (Abb. 1). Die Liebeshandlung bleibt auffallend zurückhaltend. Wenn es jedoch um die direkte Beziehung der beiden Liebenden geht, ist das Thema Schule praktisch nicht vorhanden. Sie spielt sich in sechs Szenen ab: 1. sie treffen sich zufällig in einer Kneipe, 2. sie sieht ihn zufällig auf dem Tennisplatz, 3. sie treffen sich zufällig vor dem Schultor, 4. sie passt ihn auf dem Tennisplatz ab, 5. sie haben eine Verabredung in einer Kneipe und 6. bei der finalen Szene der Brunneneinweihung akzeptiert der Direktor schließlich die Verlobung seiner Tochter, gegen die er sich die ganze Zeit gewehrt hat. Die einzige Szene, in der Dr. Kersten als Lehrer auftritt, findet während seiner ersten Stunde an der Schule statt, in welcher er durch eine ältere Nicht-Schülerin aus dem Konzept gebracht werden soll. Kurz: Der einzige Handlungsstrang von ZUR HÖLLE MIT DEN PAUKERN, der im weitesten Sinne so etwas wie eine verbindende Geschichte mit sich entwickelnden Charakteren besitzt, hat nahezu nichts mit Schule, dem eigentlichen Thema des Films, zu tun. Liebe findet nicht in der Schule statt, sondern auf dem Tennisplatz, in der Kneipe etc. (Abb. 2).

Auch zwischen Schülerinnen und Schülern sieht es in dieser Beziehung eher trübe aus. Obwohl der Schlager während der Opening-Credits $(6 \times 6=36$ von Medium-Terzett) eher die Betonung auf das jugendliche Liebesleben legt („Wer lernt, der hat viel mehr vom Leben, doch eines kann kein Buch uns geben: das Gefühl, wir sind verliebt.“), bleibt dieses zumindest für die Schülerschaft im Film gänzlich ausgespart. Neben der Romanze von Lehrer und Studentin treten Schwärmereien ausschließlich gegenüber und zwischen Erwachsenen auf: Frau Dr. Pollhagen, wie alle Frauen und Mädchen mit Sprechrolle, findet Dr. Kersten attraktiv, Pepes Vater versucht die französische Austauschschülerin $\mathrm{zu}$ verführen (Abb. 3), und auch der Kultusminister lässt sich dieser gegenüber zu halbseidenen Anspielungen und aufgeladenen Blicken herab.

Was der Film an Liebe bei den Mädchen und Jungen vermissen lässt, holt er jedoch mit Körpern wieder auf. Diese tauchen in ZUR HÖLLE MIT DEN PAUKERN vor allem als kommodifizierte und zweckhaft verdinglichte Objekte auf: $\mathrm{Zu}$ Beginn täuscht Pepe mit einem beherzten Sprung aus dem Fenster seinen heroischen Freitod vor. Der entsetzte Lehrer Prof. Knörz sieht bei dem Blick hinaus eine Attrappe des eigentlich sicher ein Stockwerk tiefer gelandeten

Schulpersonal zu, welches meist von unterschiedlichen Schauspieler`innen verkörpert wurde. Hannelore Elsner spielt zweimal die Austauschschülerin und einmal Pepes Schwester usw. 


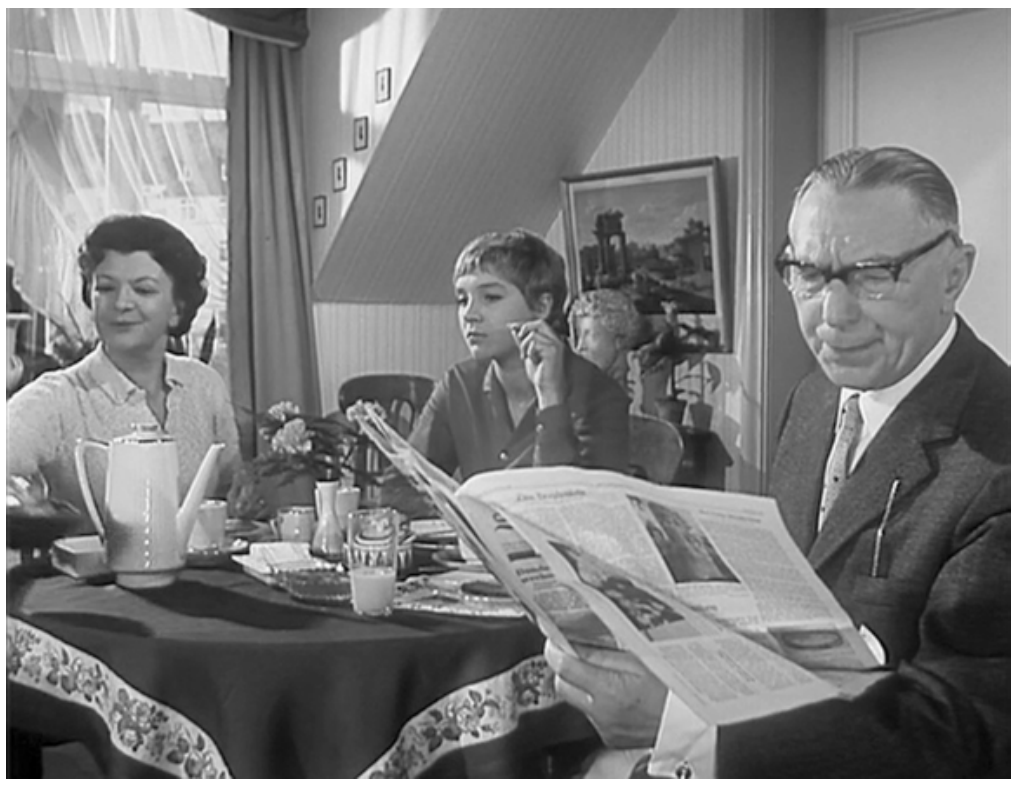

Abb. 1: Die bildungsbürgerliche Familie.

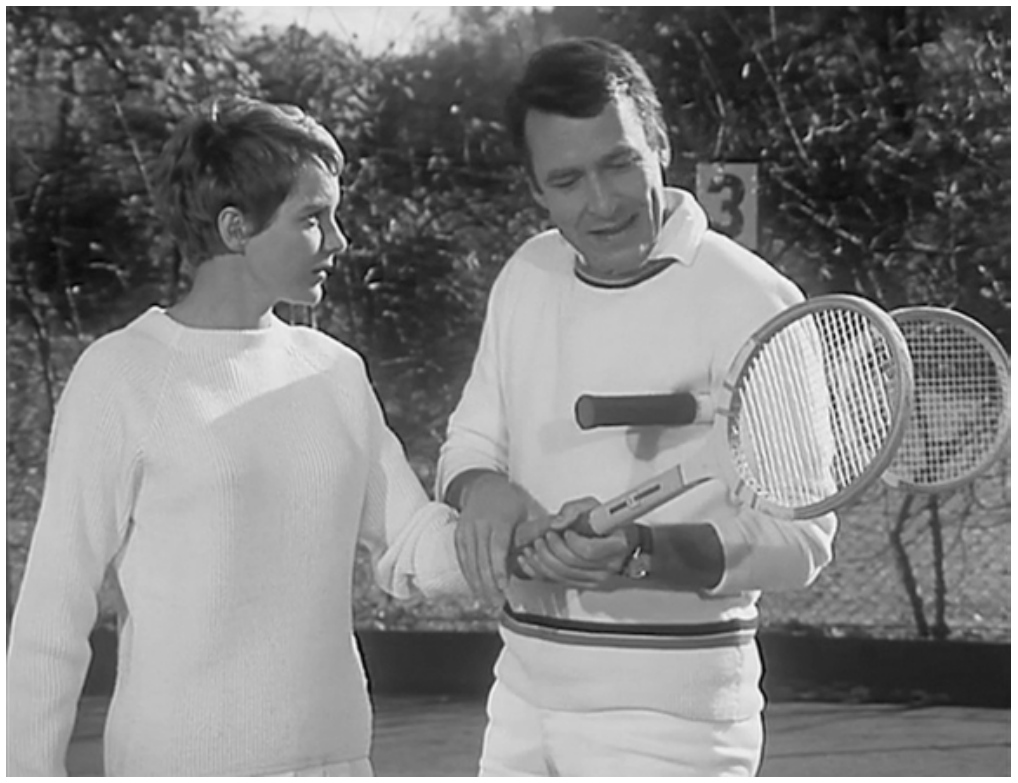

Abb. 2: Liebe auf dem Tennis Court. 
Schülers auf dem Schulhof liegen (Abb. 4). Später wird ebenfalls eine Attrappe genutzt, um der anscheinend einzigen Lehrerin der Schule vorzugaukeln, die Damentoilette sei besetzt (Abb. 5). Während sich der verdinglichte Austausch zum Zwecke des Streichs also zunächst auf männliche Surrogate stützt, werden weibliche Körper nicht ersetzt, sondern direkt kommodifiziert. Man denke an die Verkäuferin, ${ }^{13}$ welche den neuen Lehrer Dr. Kersten durch ihre ,körperlichen Reize aus der Fassung bringen soll (Abb.6) oder an die französische Austauschschülerin Geneviève, die - nur in Unterwäsche bekleidet und sich somit im natürlichen Zustand junger Französinnen befindend - den Schuldirektor bei einem Streich bloßstellen soll (Abb. 7).

Die Biologielehrerin Dr. Pollhagen dient - zusammen mit dem Mathematiklehrer Dr. Blaumeier (,Wir kommen heute zu den Kurven und dem damit im Zusammenhang stehenden konstanten Glied.“) - vor allem der Bereitstellung sexueller Innuendos und körperbezogener Slapstickeinlagen: nach dem Hinweis auf ihre ,Sextanerblase' wird, wie bereits erwähnt, die Damentoilette blockiert, sodass sie auf der Suche nach anderen Räumlichkeiten im Zeitraffer und mit sich beschleunigender, zirkusartiger Musik durch das Schulgebäude irrt. Nach einer von ihr dirigierten Gesangseinlage des Chors in der Aula bleibt Pepe an ihrer Kleidung hängen und reißt ihr diese in Folge dessen bis auf die Unterwäsche herunter, was zur allgemeinen Erheiterung aller Anwesenden beiträgt (Abb. 8).

,Die Liebe“ bleibt somit eine handlungstragende Instanz, die in der eigentlichen Institution Schule keinen Platz findet. An einen (älteren) männlichen Körper gerichtete Schwärmerei ist hingegen allen weiblichen Personen ungeachtet von Alter und Stand möglich, wohingegen sich lediglich ältere Männer für jüngere Frauen interessieren, die jedoch, soviel Anstand muss schon sein, nicht mehr zur Schule gehen - oder Französinnen sind. Für die Lehrer^innen und Schüler^innen hingegen stellt der Körper vor allem eine Projektionsfläche für körperfunktionenorientierte und sexuell konnotierte Komik dar. Hier entspinnt sich ein wechselseitiges Verhältnis zwischen Lehrer- und Schülerschaft. Erstere stellen ihre maschinenhaften, veralteten, senilen, physisch oder psychisch labilen Körper als Dispositive zur Verfügung, welche von den ,Kindern“ manipuliert, ausgetauscht oder schlichtweg ihrer verkorksten ,Natur' gemäß benutzt werden. Man denke hier z. B. an den Streich mit der Gedenkveranstaltung in der Aula - die Schüler ziehen die Lehrer wie Spielzeug an ihrer eigenen Mechanik auf - laufen tun sie dann von alleine.

13 Dargestellt von der Schwedin Christina ,Britt‘ Lindberg, die in den späten 1960er/frühen 1970er Jahren durch Erotikproduktionen (u.a. zwei Schulmädchen-Report-Filme) bekannt wurde. 


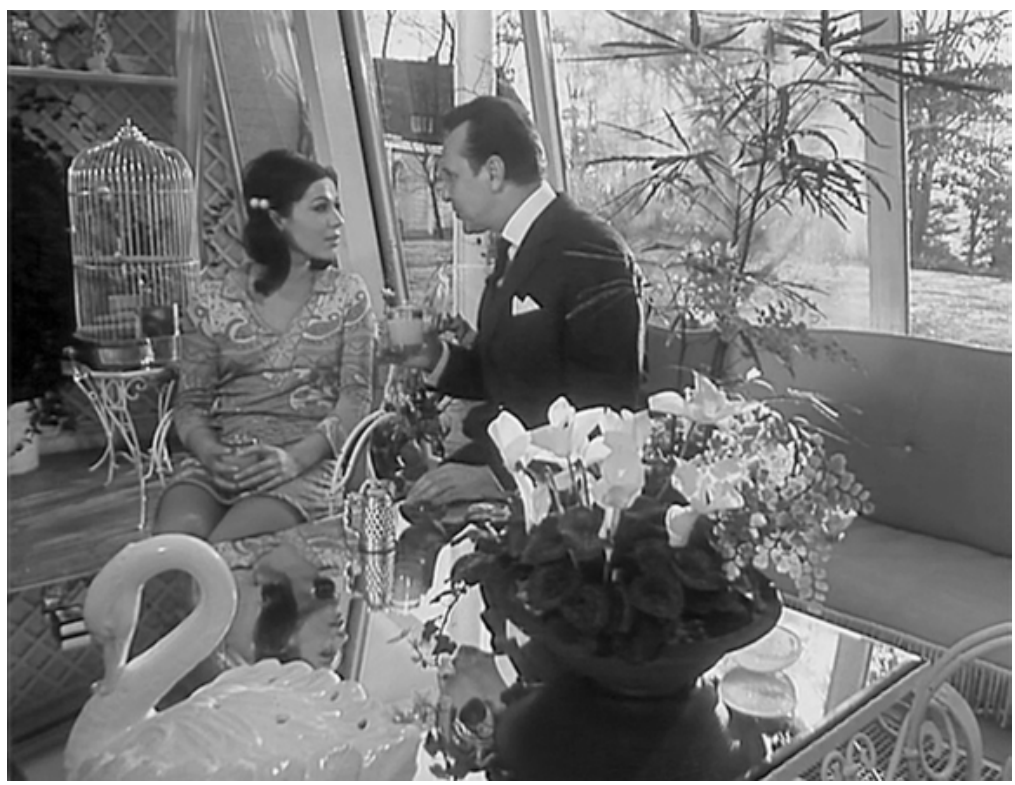

Abb. 3: Das Werben des Herrn Papa.

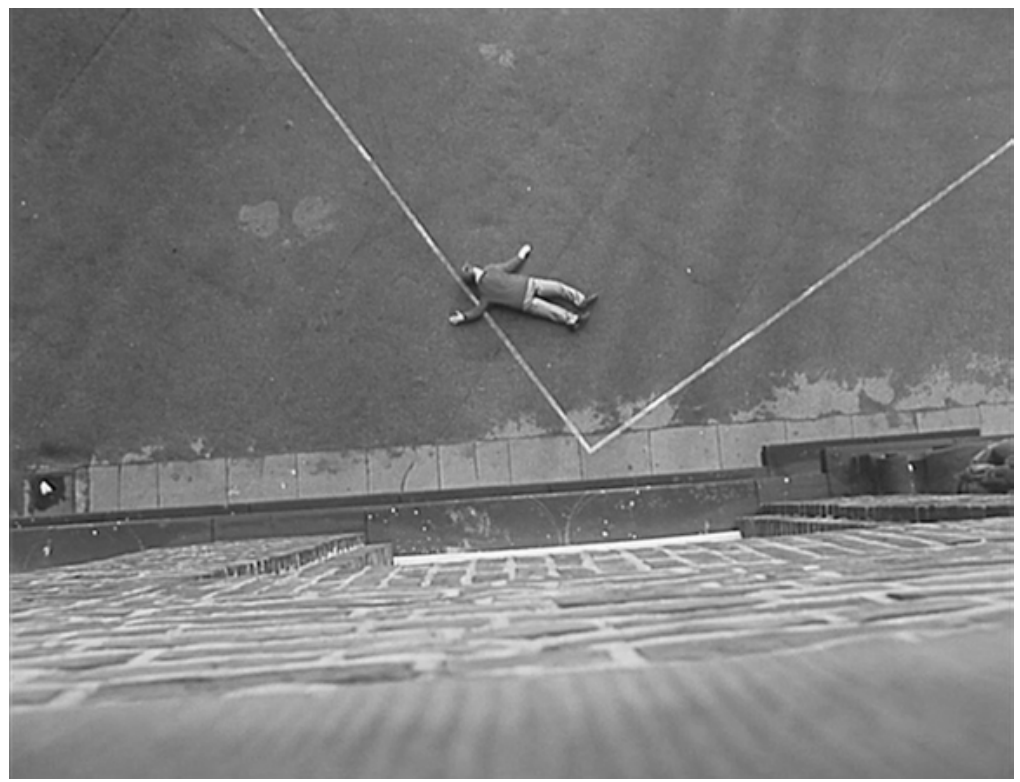

Abb. 4: Attrappe auf dem Pausenhof. 


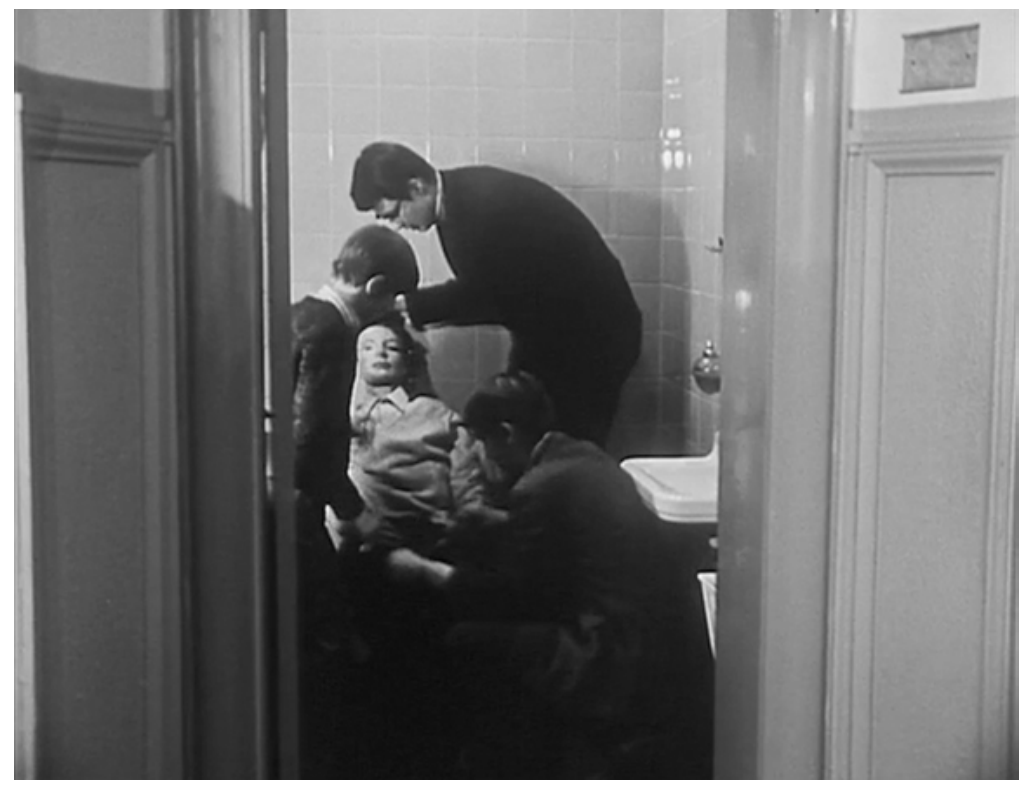

Abb. 5: Besetzt.

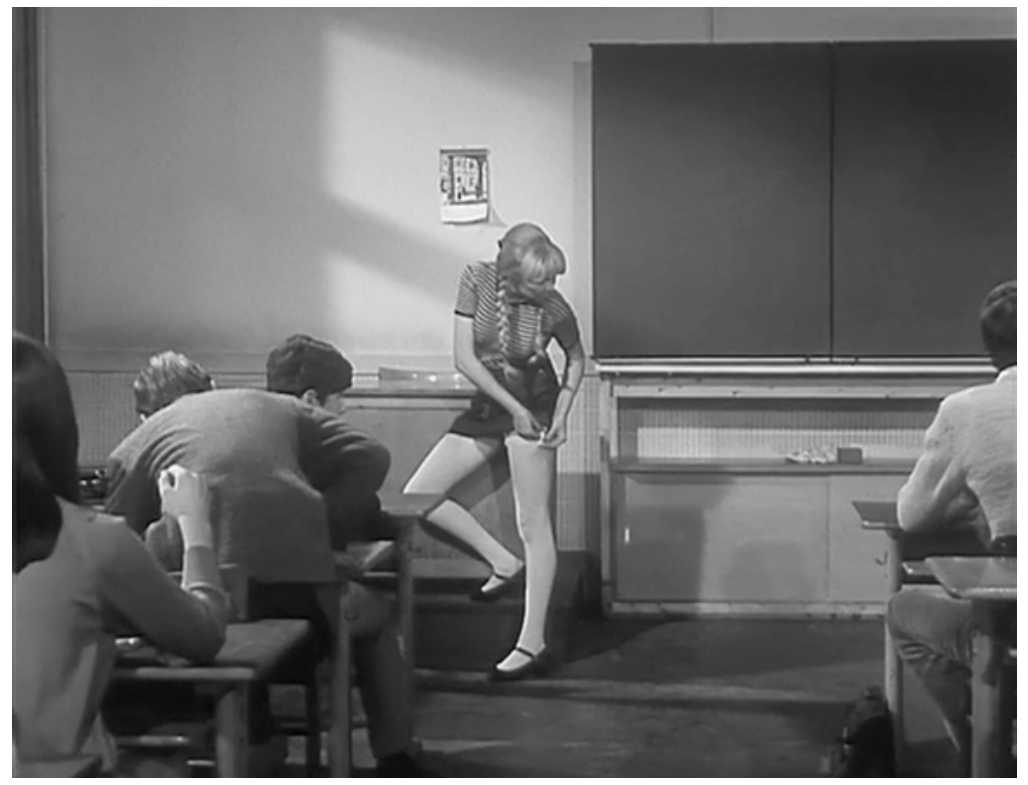

Abb. 6: Susi Rixner. 


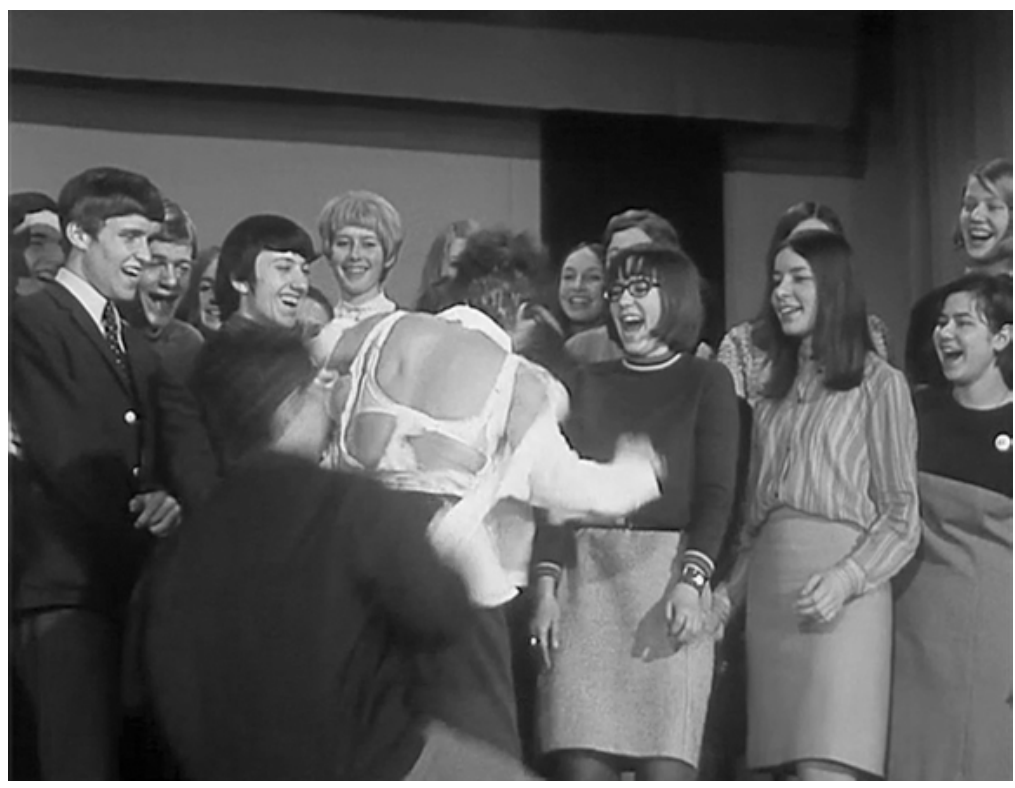

Abb. 7: Frau Dr. Pollhagen wird, entkleidet‘.

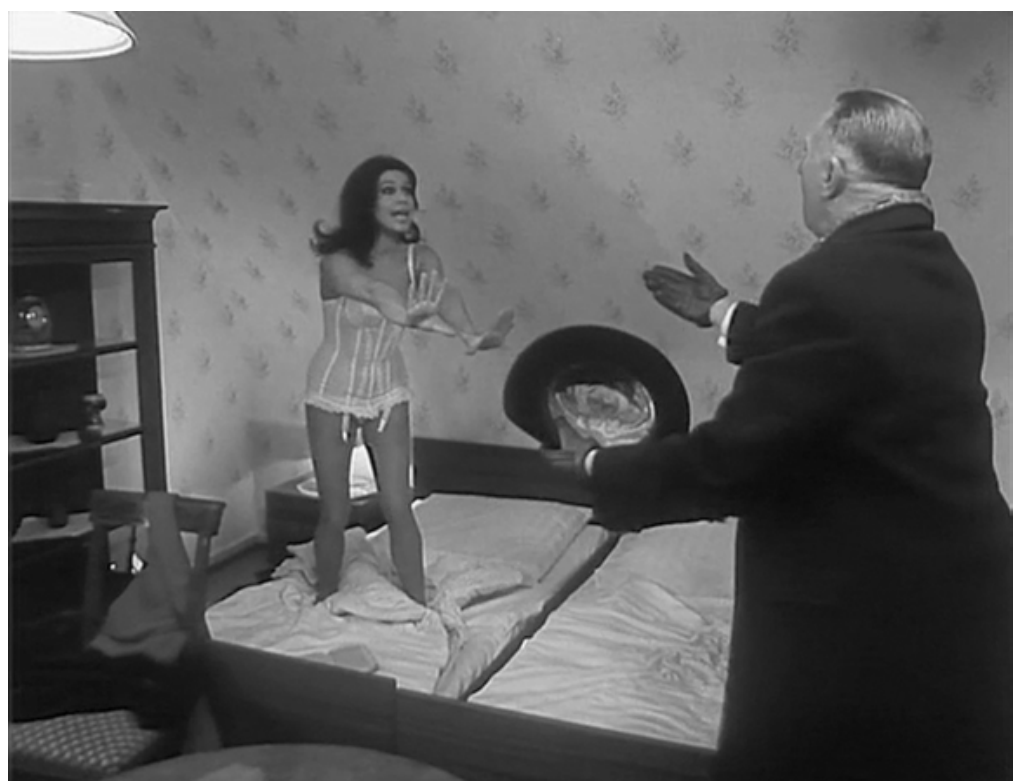

Abb. 8: Die Französin und der Herr Direktor. 


\section{Der konservative Pauker}

Eines dieser ,Pauker-Dispositive‘ lohnt es sich im Lichte der endsechziger Jahre noch einmal näher in Augenschein zu nehmen: die in ZUR HÖLLE MIT DEN PAUKERN immer präsente, vermeintliche Kritik am ewig gestrigen ,Pauker‘. Noch vor den eigentlichen Intro-Credits des Films findet dieser Dialog zwischen dem Schüler Pepe Nietnagel und dem grauhaarigen Lateinlehrer Oberstudienrat Prof. Dr. Arthur Knörz statt:

\footnotetext{
Knörz: Ach, diese Generation ist nicht wert, dass ihr Gutes getan wird.

Pepe: Warum, Herr Professor?

Knörz: Weil ihr keine Ehrfurcht mehr habt vor den nationalen Gütern und keinen Respekt mehr vor dem Lehrkörper und euren Eltern, die euch immer mit gutem Beispiel vorangegangen sind.

Pepe: Jaja, wie sie zum Beispiel Adolf Hitler gewählt haben.

Knörz: Nietnagel, Du unverschämter Kerl. Ich werde meinen ganzen Einfluss geltend machen, dass Du dieser Schule verwiesen wirst. Verlass Dich darauf.
}

Der ganze Film ist gespickt mit satirisch anmutenden ,Seitenhieben' auf die militaristische Gesinnung des älteren Lehrkörpers - die Eltern, wie in diesem Beispiel, sind eher die Ausnahme. Studienrat Dr. Blaumeier kommentiert, als er das Fehlen Nietnagels bei der Klassenarbeit bemerkt: „Aha. Das kennt man schon! Im Krieg sind Simulanten unerbittlich hingerichtet worden!“ Und Studienrat Priehl wird im Vorspann mit den Worten vorgestellt: „Der Deutsche sucht eher den Tod, als dass er seine Ehre preisgibt!“ (Pepe wiederholt diese Worte später vor seinem vermeintlich suizidalen Sprung aus dem Fenster.) Diese Lehrer sind durch ein permanent konservatives und militaristisches Auftreten geprägt.

Zurück zum erwähnten Dialog. Die Zuschreibung, dass die Eltern Hitler gewählt hätten, ist hier die zum Witz verkommene, lapidar abgeflachte und dabei entwertete Anklage eines Aspektes der Nachkriegsgesellschaft, welcher von den 1968ern kritisiert wurde. Das Problem mit diesem kurzen Dialog ergibt sich deshalb nicht aus seiner vorgeblich kaltschnäuzigen Offenbarung unausgesprochener Wahrheiten, sondern in seiner diskursiven Substanzlosigkeit. Ein anderes Beispiel: In Kurt Hoffmanns DAS SPUKSCHLOSS IM SPESSART von 1960 fahren die Geister der längst verstorbenen Spessartbanditen nach Bonn, um den Leuten von heute zu zeigen, dass es in der Hauptstadt Gespenster gibt - so auch der Text des zu hörenden Liedes. Auf ihrer Tour entfernen die unsichtbaren Wohltäter Panzer und Raketen aus einem Spielzeugladen, um sie mit Plüschtieren zu ersetzen, zerstreuen die Pension eines Generals a.D. im Winde, ändern Preise und entkleiden Schaufensterpuppen in Uniform. Der Ab- 


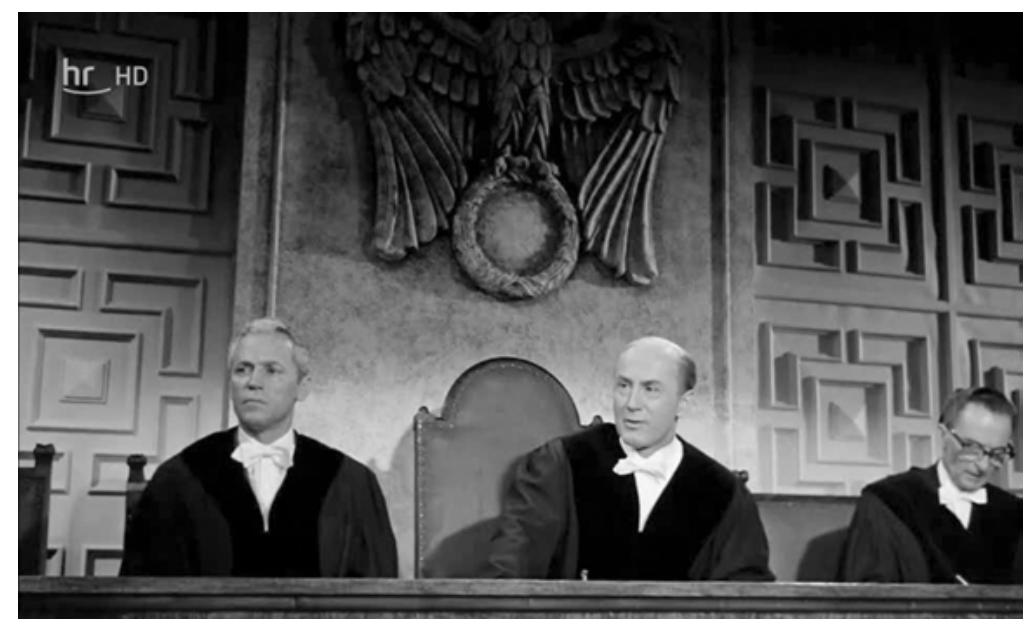

Abb. 9: „Es gibt hier keine Gespenster!“

schluss dieses Streifzuges findet in einem Gerichtssaal statt, in welchem die zu Unrecht des Diebstahls beschuldigte Gräfin (Liselotte Pulver) von einem aalglatt gescheitelten und mit Schmiss gezeichneten Richter verhört wird. Auf ihr Insistieren, dass sie unschuldig und alles eine Tat von Gespenstern sei, antwortet der Vorsitzende mit dem erbosten Ausruf „Es gibt hier keine Gespenster!“ und schlägt mit der Faust auf den Tisch. Daraufhin bröckelt im Hintergrund der Putz aus dem mit einem Adler gekrönten Eichenkranz heraus und ein Hakenkreuz kommt zum Vorschein (Abb. 9 und Abb. 10).

Doch was hat dies mit den Lümmeln zu tun?

Zum einen zeigt Hoffmanns Film, dass es sehr wohl bereits früher möglich war, jene Kritik zu äußern, auf die sich ZUR HÖLLE MIT DEN PAUKERN angeblich so augenzwinkernd beruft; zum anderen, dass es in einem Unterhaltungsfilm möglich war. DAS SPUKSCHLOSS IM SPESSART besitzt jedoch nicht die kritische Ambition, aus seinem Statement eine praktische Konsequenz zu ziehen. Bereits in der darauffolgenden Szene ist das Hakenkreuz wieder unterm Putz verschwunden; schlafend wartend auf eine Zeit, in der seine Entblößung nicht nur staunende Betroffenheit, sondern politisches Handeln auslösen würde. Es wurde genauso eingemauert wie zu Beginn des Films die Banditen des Spessarts. Und genauso kehren die Geister der Vergangenheit, die nie wirklich verschwunden waren, zurück. Jene Gespenster in Bonn sind natürlich nicht die singenden, lachenden Vagabunden, sondern die Überbleibsel von Militarismus und Faschismus, wie sie die Adenauerzeit im Gewächshaus des Vergessenwollens hat wachsen und gedeihen lassen. 


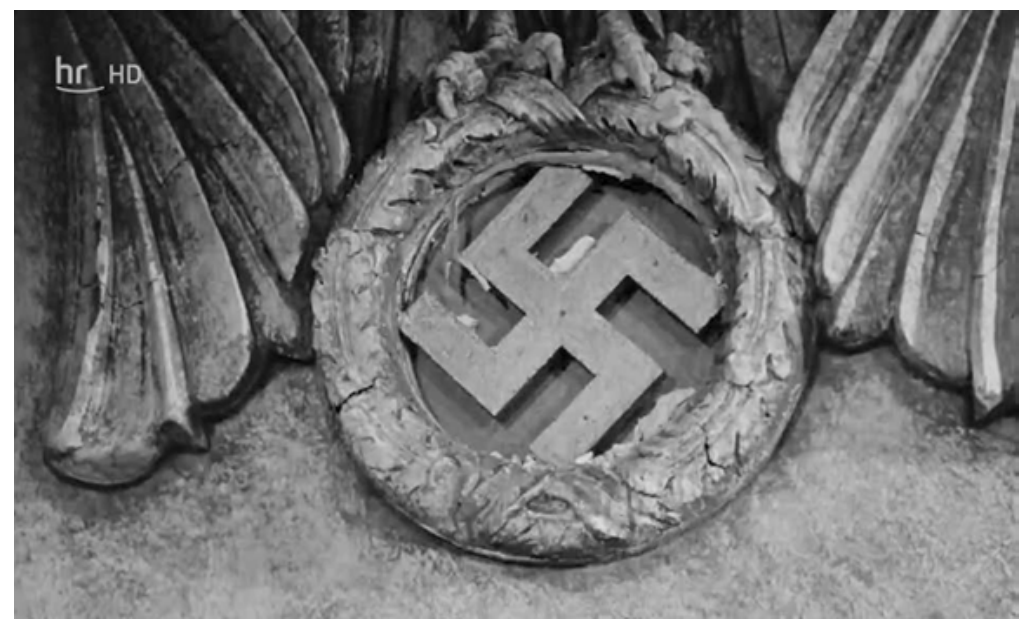

Abb. 10: Eingemauert und vergessen.

Die reaktionären, militaristischen Marotten der Lehrer in ZUR HÖLLE MIT DEN PAUKERN bleiben schrullige Charakterzüge. Sie stehen auf einer Stufe mit lustigen Dialekten, unbeabsichtigten Innuendos und allgemeiner Begriffsstutzigkeit, welche nicht - wie in DAS SPUKSCHLOSS IM SPESSART - auf eine strukturkritische Tiefe hinabreichen können. Sie führen, und hier sind sich Hoffmanns und Jacobs Film wieder ähnlich, nicht $\mathrm{zu}$ entsprechenden diskurskritischen Konsequenzen (falls sich eine solch mächtige Begrifflichkeit hier überhaupt anzubringen lohnt). Die Streiche Pepes, welche eben diese Marotten zum Angriffspunkt nehmen, gelangen in ihrer Tragweite niemals über die räumlichen Grenzen des Schulgebäudes oder die ihrer eigenen Dauer hinaus. Sie verbleiben in der filmischen Echokammer der Szene, wo sich ihre Druckwellen verstärken und schließlich wieder verschwinden - ohne jemals das Außen zu erreichen oder Auswirkungen sichtbar werden $\mathrm{zu}$ lassen. ${ }^{14}$

14 Der erste Streich treibt den alten Lehrer Prof. Knörz zwar ins Sanatorium und den neuen Dr. Kersten in die Schule, wird aber nie aufgeklärt oder inhaltlich wieder aufgegriffen. Im zweiten Streich wird die Lehrertoilette blockiert, was zu einem Slapstickauftritt Frau Dr. Pollhagens führt, der sich jedoch im Nichts verliert - sie verschwindet im Treppenhaus. Um den neuen Lehrer Dr. Kersten zu ärgern, wird die Verkäuferin Susi Rixner für den dritten Streich engagiert. Der Lehrer - als progressiver und mit allen Wassern gewaschener Junglehrer - fällt jedoch nicht darauf herein. Dies ist seine erste und im Film auch einzige Unterrichtsstunde er verbringt mehr screen-time auf dem Tennisplatz als im Schulzimmer. Der vierte Streich führt nach einer manipulierten Lautsprecherdurchsage zu einer ungewollten Aulaveranstaltung er bleibt ohne Konsequenz und Tragweite -, und im fünften Streich wird ein Luftalarm vorgetäuscht; was im totalen Chaos endet, ohne jedoch jemals wieder erwähnt zu werden. Streiche 
Der Militarismus und das Überwintern nationalsozialistischer Personalien und Einstellungen in der Gesellschaft der jungen Bundesrepublik werden hier nicht kritisiert, sondern als verschrobene Attitüde weltfremder Pauker ohne gesellschaftspolitischen Einflussbereich inszeniert. Darüber mag man lachen, aber empören kann man sich nicht. Im Gegenteil, die eigentliche Kritik der endsechziger Jahre wird hier für eine Komik verramscht, die den eigentlichen Diskurs desaströs verharmlost.

Im Zuge dessen muss jedoch auch auf eine andere Verknüpfung dieser spezifischen Attitüde der Filme hingewiesen werden. Über die Produktionsfirma und den Protagonisten ergeben sich Parallelen zu den Lausbubenfilmen mit Hansi Kraus, dessen erster Teil, Helmut Käutners LAUSBUBEnGESCHICHTEN (BRD 1964) nach der literarischen Vorlage von Ludwig Thoma, ebenso episodischen Charakter wie ZUR HÖLLE MIT DEN PAUKERN aufweist. Auch teilen sich beide Serien immer wieder die gleichen Schauspieler, sind innerhalb ihrer eigenen Reihe ebenso verwoben wie unverbunden und nutzen die gleiche chargenhafte Zielscheibe: Preußen. Das, was sich in ZUR HÖLLE MIT DEN PAUKERN als Entblößung reaktionärer und militaristischer Tendenzen des Lehrkörpers geriert, ist im Grunde nichts anderes als die Verballhornung einer klischeeüberfrachteten Vorstellung von preußischem Beamten- und Soldatentum. Konsequenterweise muss man diese semiotischen Überschneidungen süddeutscher Possen- und Schwankmotive mit in Rechnung ziehen, wenn man versucht, sich von dem eigentümlichen Ding eine Vorstellung zu machen, das ZUR HÖLLE MIT DEN PAUKERN ist.

\section{Das affektive Zentrum imaginierter Zeitlosigkeit}

Mit den bisher gemachten Beobachtungen möchte ich die Vorstellung des ,affektiven Zentrums‘ präzisieren, das sich um das Konglomerat von Paukerfilmen, Schlagermusik und Heimatmotiven gebildet hat. Dieses Zentrum beschreibt dabei weniger eine inhaltliche oder dramaturgische Dimension, sondern eher eine spezifische raumzeitliche Weltentfaltung, die sich in den oben beschriebenen

sechs und sieben hängen zusammen und sind die einzigen mit inhaltlicher Konsequenz. Pepe setzt eine falsche Heiratsanzeige für Dr. Kersten und Helena - die Tochter des Rektors - auf und fingiert, nachdem dies nicht die gewünschte Wirkung erzielte und der Rektor die Beziehung seiner Tochter akzeptierte, ein verfängliches Treffen mit dem Rektor und der Austauschschülerin. Auch dieses platzt im letzten Moment. Der letzte Streich lässt den Brunnen im Schulhof bei seiner Einweihung in Pyrotechnik aufgehen. Damit endet der Film. 
Aspekten von Episodenhaftigkeit, Körperbild und chargenhafter Typisierung abzeichnet.

Obwohl es Titel und Thema suggerieren mögen, geht es in ZUR HÖLLE MIT DEN PAUKERN nur scheinbar um jene ,Penne‘, die sich im geteilten Gedächtnis als seltsam nostalgischer, halb gefürchteter, halb romantisierter Ort verwegener Abenteuer und skurriler Lehrer manifestiert. Der Film hat weit mehr mit dem süddeutschen Heimatschwank à la LAUSBUBENGESCHICHTEN und seiner derben, körperbetonten Komik, der Verballhornung des Preußentums und der Fixierung auf eine verklemmt abwesende Erotik zu tun, mit der Posse und ihren in der Episode eingekapselten artistischen Kapriolen, mit dem Heimatfilm und seiner Zentrierung auf die zu Anfang durch Standesgrenzen unüberbrückbar scheinende, aber dann doch ermöglichte Liebesbeziehung ${ }^{15}$ und dem Schlagerfilm mit seinen - nun ja - Schlagern. Dieser Film, wie viele in seinem Gefolge, ist jedoch kein wüster Genremix oder Hybridfilm, sondern Ausdruck einer sehr spezifischen, räumlich-zeitlichen Verfasstheit, welche den Moment der Schule aus seiner Verankerung in der Gegenwart herauslöst und in den Erfahrungsbereich einer imaginierten Zeitlosigkeit versetzt. Dies ist das ,affektive Zentrum' um das dieser Film und das ganze Konglomerat an Pauker-, Penne- und Lümmelfilmen kreist. Es vereint eine spezifische Erfahrung, welche sich nicht auf eine konkrete, sondern verklärte ,Erfahrbarkeit von Welt' beruft, die ausschließlich im Jetzt stattfindet und keine Anschlüsse an eine unmittelbare Zukunft kennt, sondern lediglich den Weg zurück, in die nostalgische Verschleierung des Augenblicks.

Das Geschehen von ZUR HÖLLE MIT DEN PAUKERN findet in einer Art zeitlosen Suspendierung statt. Bereits zu Beginn erscheint Pepe Nietnagel und stellt die Lehrenden in einer Ansprache direkt in die Kamera vor. Sie sind dabei in Farb- und Schwarzweiß-Fotos oder kurzen Ausschnitten zu sehen, von denen einige später im Laufe des Films noch einmal auftauchen werden, andere nicht. Obwohl das Schulgeschehen per Definition im curricularen Progress aus Prüfungen, Zeugnissen, Ferien usw. besteht, gibt es hier im Schuljahr keinen Anfang und kein Ende. Alles findet in einem kalendarischen Vakuum statt, das weder beginnt noch endet, in dem es immer nur Klassenarbeiten, aber nie Zeugnisse gibt und niemand die Ferien auch nur erwähnt. Diese Schule ,in Limbo' findet sich bereits in Helmut Weiss' dIE FEUERZANGENBowle (D 1944) und charakterisiert eine ganz spezifische ästhetische Verfasstheit - jene des träumerischen Rückzugs aus dem Jetzt. Sie beschreibt eine Welt, die aufgehört hat sich zu drehen und in einem Status Quo verharrt, welcher die ästheti-

15 Vgl. Koch et al.: Die fünfziger Jahre, S. 84. 
schen Modi immer nur wieder perpetuieren kann, ohne sich von ihnen zu lösen oder sie zu verarbeiten. Selbst Filme wie Alex von Ambessers DER PAUKER (BRD 1958), DER MUSTERKNABE oder ... UND SOWAS MUSS UM 8 INS BETT sind an einen räumlich-zeitlichen Horizont gekoppelt, der sie in einer Erfahrung des Jetzt situiert. Die Lümmelfilme sind jedoch viel eher im zeitlichen Modus des Heimatfilms zu finden als in jenem anderer Schulfilme. Dies bezeichnet dabei weniger das narrative Setting als eine Art zeitlicher Entrückung. Ines Steiner beschreibt in ihrer Analyse des Heimatfilms der 1960er Jahre, wie seine Formen nicht untergegangen sind, sondern immer wieder mit veränderten Gesichtern im Fernsehen auftauchen, sei es in der Lindenstraße oder der Schwarzwaldklinik:

Doch wesentlicher erscheint, daß das Fernsehen damit in zeitgenössisch kostümierter Gestalt die aus den 50er-Jahre-Streifen bekannten geschlossenen Weltbilder reproduziert, die Sicherheit in der Überschaubarkeit anbieten und Krisenmanagement durch den Rat von Autoritäten, von neuen alten Vaterfiguren wie dem Arzt und dem Pfarrer versprechen. ${ }^{16}$

Abgesehen vom narrativen Inhalt kann man hier sagen, dass sich die Lümmelfilme und ihre Ableger unterschiedlichster Couleur durch eine die Gegenwart suspendierende zeitliche Erfahrung auszeichnen, welche sich am ehesten mit der ,heilen Welt' des Heimatfilms und ihren belanglosen und immer lösbaren Problemen beschäftigt, ohne ein Heimatfilm zu sein. Die Schule ist hier kein traumhafter Ort, der irgendwo existiert, sondern ein zeitlich entrücktes Surrogat, das nie wirklich da war. Die episodische Struktur der Filme, wie auch die permutierende Bezugnahme auf Vorgänger, Nachfolger und Nachahmer steigert dabei die alte Anklage an das deutsche Nachkriegskino, stets nur eine Wiederholung des Immergleichen zu sein, ins Groteske.

Gaudeamus igitur, jenes Studentenlied, welches auf musikalischer Ebene in praktisch allen diesen Filmen zu finden ist - jedoch nie intoniert wird -, besingt übrigens die Schönheit des Augenblicks und die Erinnerung an Freunde, Akademie und Lehrkräfte in Anbetracht des Wissens, dass wir alle dereinst sterben werden.

16 Thomas Hoffmann/Ines Steiner: Die sechziger Jahre. Zwischen Jagdszenen und Jägerporno. In: Kaschuba, Wolfgang (Hg.): Der deutsche Heimatfilm. Bildwelten und Weltbilder - Bilder, Texte, Analysen zu 70 Jahren deutscher Filmgeschichte. Tübingen 1989, S. 97-129, S. 102. 


\section{Literaturverzeichnis}

Bergfelder, Tim: Exotic Thrills and Bedroom Manuals. West German B-Film Production in the 1960s. In: Randall Halle/Margaret McCarthy (Hg.): Light Movies. German Popular Film in Perspective. Detroit 2003, S. 197-219.

Grob, Norbert: Film der sechziger Jahre. Abschied von den Eltern. In: Wolfgang Jacobson/ Anton Kaes/Hans Helmut Prinzler (Hg.): Geschichte des deutschen Films. Stuttgart/ Weimar 2004, S. 207-244.

Hoffmann, Thomas/Steiner, Ines: Die sechziger Jahre. Zwischen Jagdszenen und Jägerporno. In: Wolfgang Kaschuba (Hg.): Der deutsche Heimatfilm. Bildwelten und Weltbilder Bilder, Texte, Analysen zu 70 Jahren deutscher Filmgeschichte. Tübingen 1989, S. 97129.

Koch, Friedrich: Schule im Kino. Autorität und Erziehung. Vom ,Blauen Engel‘ bis zur ,Feuerzangenbowle‘. Weinheim/Basel 1987.

Koch, Gertraud/Konz, Klaus/Oehrle, Wolfgang/Schmidt, Gundula/Wilzcek, Barbara: Die fünfziger Jahre. Heide und Silberwald. In: Wolfgang Kaschuba (Hg.): Der deutsche Heimatfilm. Bildwelten und Weltbilder - Bilder, Texte, Analysen zu 70 Jahren deutscher Filmgeschichte. Tübingen 1989, S. 69-95.

Kramp, Joachim: Die Lümmel von der ersten Bank: Die Lümmel sind los! Auf: Luemmelbank.blogspot.de: http://luemmelbank.blogspot.de/p/die-lummel-sind-los.html (27. 03. 2018).

Maase, Kaspar: Was macht Populärkultur politisch? Wiesbaden 2010.

Wulff, Hans J.: Hybridität der Gattungen. Schlagerfilm / Filmschwank / Schlagerfilmschwank. In: Hanno Berger/Frédéric Döhl/Thomas Morsch (Hg.): Prekäre Genres. Zur Ästhetik peripherer, apokrypher und liminaler Gattungen. Bielefeld 2015, S. 217-235.

o. V.: Jahrescharts Deutschland 1968 Auf: chartsurfer.de: http://www.chartsurfer.de/musik/ single-charts-deutschland/jahrescharts/hits-1968-2x1.html (27. 04. 2018).

o. V.: Die erfolgreichsten deutschen Filme seit 1963 (2018). Auf: insidekino.de: http://www.insidekino.com/DJahr/DAlltimeDeutsch50.htm (27. 04. 2018).

\section{Filmografie}

... UND SOWAS MUSS UM 8 INS BETT. Reg. Werner Jacobs. BRD 1965.

DAS SPUKSCHLOSS IM SPESSART. Reg. Kurt Hoffmann. BRD 1960.

DER MUSTERKNABE. Reg. Werner Jacobs. BRD 1963.

DER PAUKER. Reg. Alex von Ambesser. BRD 1958.

Die feuerzangenbowle. Reg. Helmut Weiss. D 1944.

DIE HEIDEN VON KUMMEROW UND IHRE LUSTIGEN STREICHE. Reg. Werner Jacobs. BRD/DDR 1967.

HURRA, DIE SCHULE BRENNT! Reg. Werner Jacobs. BRD 1969.

LAUSBUBENGESCHICHTEN. Reg. Helmut Käutner. BRD 1964.

PEPE, DER PAUKERSCHRECK. Reg. Harald Reinl. BRD 1969.

UNSERE PAUKER GEHEN IN DIE LUFT. Reg. Harald Vock. BRD 1970.

WIR HAU'N DIE PAUKER IN DIE PFANNE. Reg. Harald Reinl. BRD 1970.

ZUM TEUFEL MIT DER PENNE. Reg. Werner Jacobs. BRD 1968.

ZUR HÖLLE MIT DEN PAUKERN. Reg. Werner Jacobs. BRD 1968. 